\title{
Intracranial abscess from facial cellulitis
}

\author{
Jonghyun Park, \\ Woo Seob Kim, \\ Han Koo Kim, \\ Tae Hui Bae \\ Department of Plastic and \\ Reconstructive Surgery, Chung-Ang \\ University Hospital, Chung-Ang \\ University College of Medicine, Seoul, \\ Korea
}

\begin{abstract}
Cellulitis, one of most common diseases of everyday life, is often overlooked for its significance. Although cellulitis does not cause or lead to serious problems usually, its possibility to cause lifethreatening problem should be known. In present case, a patient who had received acupuncture treatment a week earlier presented to the clinic with symptoms of facial cellulitis. The disease resolved within few weeks under empirical antibiotic treatment but recurred after 3 months. Under close history review of the patient, we found out that the patient had received craniectomy 20 years ago. The patient had blunt headache with no other neurological symptoms that could suspect cranial infection, but considering the risk originating from the patient's surgical history, brain computed tomography (CT) was taken. CT images revealed abscess formation in the subgaleal and epidural spaces. Craniotomy with abscess evacuation was done promptly. With additional antibiotic treatment postoperatively, the disease resolved, and the 1-month postoperative followup brain CT showed no signs of abscess formation.
\end{abstract}

Keywords: Acupuncture / Cellulitis / Empyema / Epidural abscess

\section{INTRODUCTION}

Infection is defined as an invasion or multiplication of microorganisms that are not normally present within the body. Individuals can experience infections as simple as cellulitis to those as lethal as intracranial empyema in everyday life. Cellulitis is one of the most common diseases that physicians encounter in clinics and is also one disease that could be overlooked for its significance. Although the lower extremities are the most common site of cellulitis, facial cellulitis remains frequent, accounting for $4 \%$ to $24 \%$ of all cellulitis cases [1]. When facial cellulitis is suspected, there are several diseases to consider in the differential diagnosis; however, intracranial empyema is not commonly considered [2]. Intracranial empyema, located in epidural or subdural spaces, usually presents with a triad of symptoms: fever, headache, and vomiting [3].

\section{Correspondence: Woo Seob Kim}

Department of Plastic and Reconstructive Surgery, Chung-Ang University

Hospital, 102 Heukseok-ro, Dongjak-gu, Seoul 06973, Korea

E-mail: kimws@cau.ac.kr

Received July 24, 2019 / Revised August 19, 2019 / Accepted September 18, 2019
In the present case, a patient previously diagnosed with facial cellulitis, with erythematous swelling on his right cheek, was later diagnosed with epidural empyema. The patient did not exhibit the most common triad of symptoms of epidural empyema; however, epidural abscess was revealed on radiological imaging and surgical drainage was performed.

\section{CASE REPORT}

The patient described in this report provided written consent for publication of anonymized case details and images. A 77-year-old male patient was admitted to the emergency department for swelling in the right cheek and temporal areas. He underwent acupuncture therapy 1 week previously on his right cheek for occasional headache, which the patient had over the past several years. On physical examination, he exhibited erythematous skin color, mild heat, and tenderness (Fig. 1). There were no signs of fluctuation or pus formation. Laboratory results revealed a C-reactive protein (CRP) level of $96.3 \mathrm{mg} / \mathrm{L}$, a white blood cell (WBC) count of $8.95 \times 10^{9} / \mathrm{L}$, neutrophil per- 


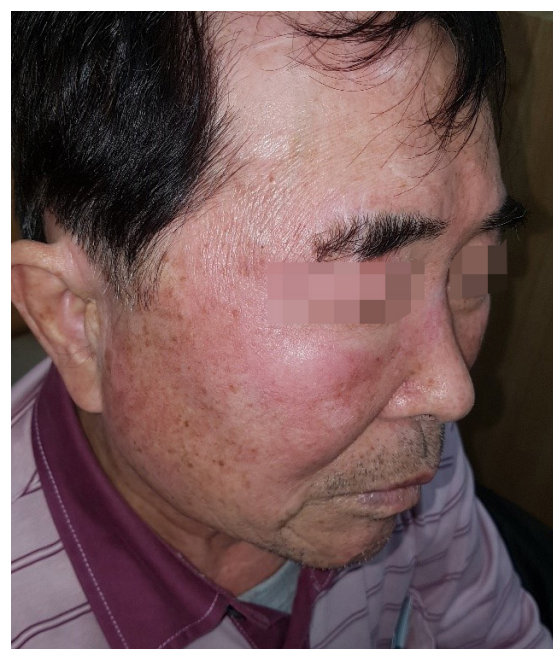

Fig. 1. Erythematous swelling on the right cheek.

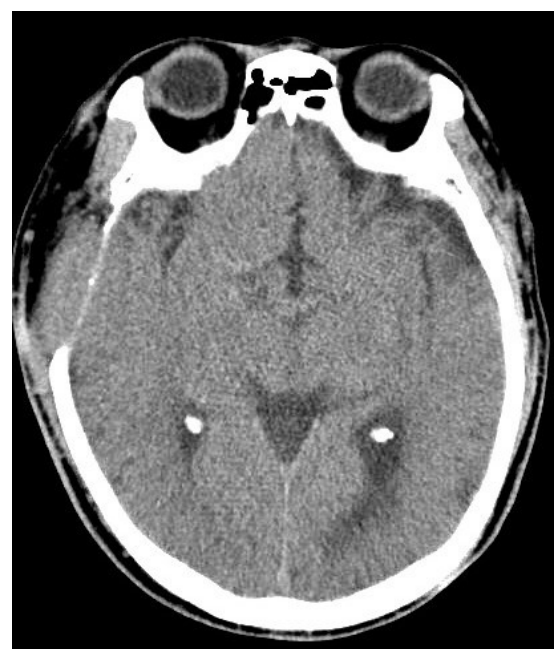

Fig. 2. A bone defect in the right parietal area.
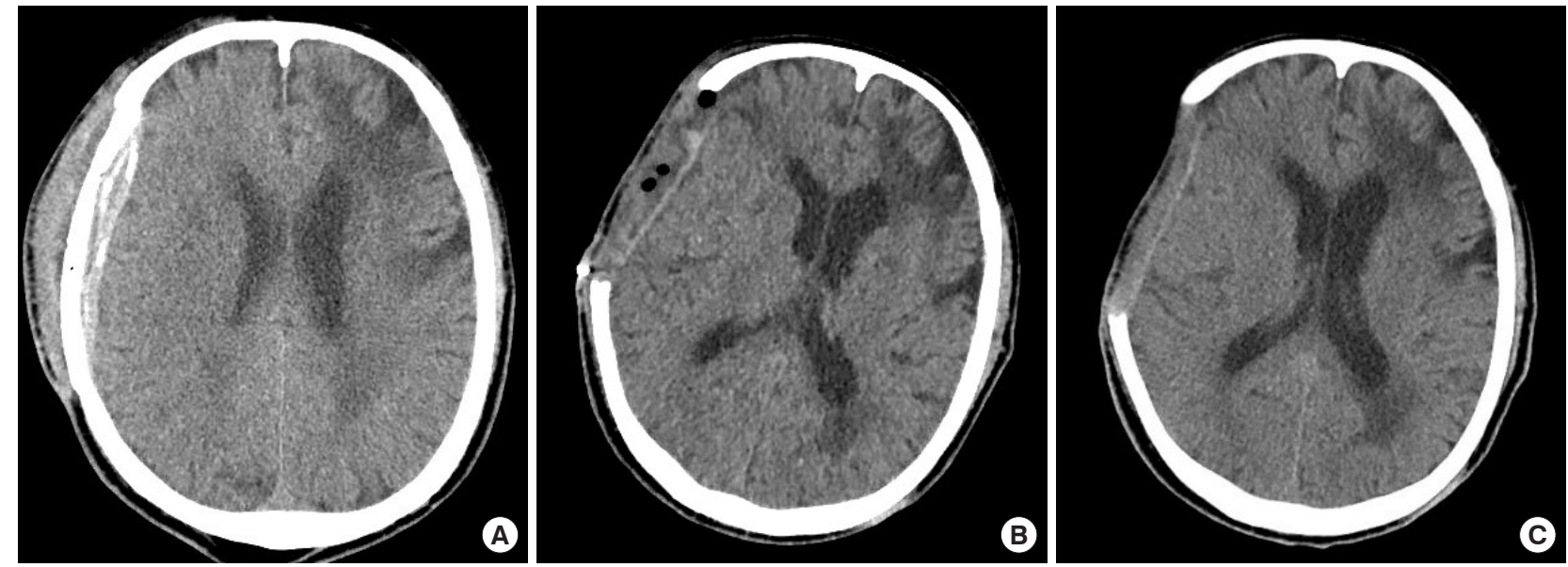

Fig. 3. (A) Preoperative brain computed tomography (CT) with heterogeneous fluid collection in the subgaleal and epidural spaces. (B) Immediate postoperative brain CT. (C) One month postoperative brain CT.

centage of $81.9 \%$, and erythrocyte sedimentation rate (ESR) of $25 \mathrm{~mm} / \mathrm{hr}$. The patient was diagnosed with cellulitis and underwent intravenous antibiotic therapy with cefazolin. One week later, laboratory results improved (CRP, $2.0 \mathrm{mg} / \mathrm{L}$; ESR, $7 \mathrm{~mm} /$ hr), with no signs of infection on physical examination. After an additional week of therapy, treatment concluded.

The patient returned 3 months after his last visit complaining of recurrent cellulitis. On physical examination, the patient exhibited erythematous swelling in the same area. Laboratory investigations revealed a CRP level of $72.7 \mathrm{mg} / \mathrm{L}$, a WBC count of $10.5 \times 10^{9} / \mathrm{L}$, and an ESR of $39 \mathrm{~mm} / \mathrm{hr}$. In addition, the patient complained of blunt headache in the right temporal area with no other neurological symptoms. On further history taking, the patient reported that he sustained a cranial injury 20 years previously and had undergone a craniectomy. Given the patient's surgical history, brain computed tomography (CT) was prompt- ly performed considering the risk for brain infection.

CT revealed a bone defect in the right parietal area of the skull from the previous surgery (Fig. 2). There was heterogeneous fluid collection in the subgaleal and epidural spaces, with right cerebral convexity and dural thickening and enhancement. There was no significant midline shifting of the brain (Fig. 3A). He was started on ceftriaxone as empirical broad-spectrum therapy, and was transferred to the operating room for source control and specimen sampling as soon as general anesthesia was available. An incision was made on the scar from the previous craniectomy and an additional craniotomy was performed adjacent to the bone defect to approach the suspicious infection source. Purulent fluid collection was present in the subgaleal and epidural spaces, which was sent for culture of aerobic and anaerobic organisms. All purulent collection was removed, and massive irrigation was performed with povidone-iodine solu- 
tion and normal saline. The cutaneous flap was closed and a drain was left in situ.

Postoperative brain CT revealed no evidence of residual fluid collection (Fig. 3B). The patient's laboratory results 6 days postoperatively improved (CRP, $0.5 \mathrm{mg} / \mathrm{L}$; WBC, $3.75 \times 10^{9} / \mathrm{L}$ ), with no clinical complications.

The specimens sent for culture grew Staphylococcus aureus and the patient underwent additional 3 weeks of intravenous antibiotic treatment before being discharged.

On clinical and radiological follow-up (1 month after surgery), there were no neurological symptoms or complications, and CT revealed complete resolution of the disease (Fig. 3C).

\section{DISCUSSION}

Patients with facial cellulitis, unlike other forms of cellulitis, are usually hospitalized due to the perceived risk for complications. Although the incidence of cellulitis has dropped dramatically since the inception of the antibiotic era, complications still occur, and the most feared complication of facial cellulitis is cerebral venous sinus thrombosis (CVT) [1]. This rare complication is more likely to occur, not in patients with facial cellulitis alone, but together with sinusitis and orbital cellulitis $[1,4]$. Cavernous sinuses are the most commonly involved sinuses in septic CVT, which usually follow the infection of paranasal sinuses, dental abscesses, otitis media, and orbital infections [5]. Cavernous sinuses are only separated by a thin bony wall or only soft tissue with adjacent sinuses. In addition, the oculomotor, trochlear, and trigeminal nerves travel adjacent to cavernous sinus, and the abducens nerve and carotid artery pass through the cavernous sinus [6]. These anatomical peculiarities play a role in making cavernous sinus susceptible to infections involving facial structures [4].

Given that cavernous sinus is presumably susceptible to facial infection, orbital cellulitis and sinusitis are also known to cause intracranial abscess [7-9]. Brain abscess is a rare and life-threatening condition that typically originates from direct extension from nearby structures, hematogenous dissemination, or following penetrating cerebral trauma or neurosurgery $[8,10]$. Diagnosing brain abscess is challenging due to its nonspecific presentations. The most common triad of symptoms include fever, headache, and vomiting; however, not all patients exhibit all common symptoms. The most common sources of brain abscess are neurosurgical procedures, and are most commonly caused by Staphylococcus and Streptococcus species [3,11]. The prognosis of patients with brain abscess critically depends on rapid diagnosis, prompt initiation of appropriate antibiotics, and early surgical intervention [3]. Usually, brain abscess caused by complications of sinusitis and orbital cellulitis can be found located in the frontal lobe of the brain [12].

In the present case, the patient did not exhibit signs of orbital cellulitis nor sinusitis, and the abscess was found near the temporal lobe. Although the patient did have a history of brain surgery, it was 20 years before the facial cellulitis and was less likely to have caused the infection. Despite the possibility of the presence of epidural abscess before the patient had received acupuncture treatment cannot be ruled out, because he has had occasional headache over the past several years, we focused on the more likely probability of recent acupuncture directly causing the epidural abscess.

Acupuncture, although reported for complications such as cellulitis, epidural abscess, septic arthritis, musculoskeletal abscess and, very rarely, renal failure, is not known to cause brain abscess $[13,14]$. It is challenging to find studies in the literature in which acupuncture caused brain abscess. Recently, Priola et al. [7] reported a rare case of acupuncture causing brain abscess in an immunosuppressed patient. Although acupuncture does not generally cause serious problems, specific risk factors, such as immunosuppression, and specific acupuncture sites, such as joint spaces, the meninges and ears, could cause complications [15]. In our case, the patient had anatomical predisposing factor of a skull defect on the right parietal area, resulting from previous craniectomy, which may have played a crucial role in causing the brain abscess from acupuncture. An interesting and alarming aspect of this case was that the patient did not initially present with common neurological symptoms, but with usual symptoms of facial cellulitis. The symptoms of facial cellulitis improved after antibiotic treatment and appeared to resolve but relapsed within 3 months.

There could be possibilities of either facial cellulitis progressing to brain abscess or brain abscess presenting as facial cellulitis. Since the cellulitis symptoms improved after antibiotic treatment on the patient's first visit, the possibilities of cellulitis aggravating to epidural abscess was considered low. On the other hand, S. aureus grew from the cultured brain abscess specimen, and empirical antibiotics used for facial cellulitis, such as cefazolin, covering Gram-positive cocci bacteria, were effective. This may have reduced superficial inflammation caused by the brain abscess and camouflaged deep-seated infection, presenting as relapsing facial cellulitis after a few months.

In conclusion, symptoms may not always reflect the actual disease. As such, facial infection, no matter how innocuous, should be treated thoroughly. Physicians should always be diligent in finding predisposing factors that could lead to serious infection such as brain abscess. Even acupuncture, which is not considered to be very dangerous, could be lethal. It would be helpful 
for patients, especially in Asian cultures where acupuncture is commonly practiced, to be wary of the side effects acupuncture may have after surgical procedures such as craniectomy.

\section{NOTES}

\section{Conflict of interest}

No potential conflict of interest relevant to this article was reported.

\section{Ethical approval}

The study was approved by the Institutional Review Board of Chung-Ang University Hospital (IRB No. 1906-009-16268) and performed in accordance with the principles of the Declaration of Helsinki. Written informed consent was obtained.

\section{Patient consent}

The patient provided written informed consent for the publication and the use of his images.

\section{ORCID}

Jonghyun Park

https://orcid.org/0000-0001-9910-6945

Woo Seob Kim https://orcid.org/0000-0002-4104-3926

Han Koo Kim https://orcid.org/0000-0002-2849-3973

Tae Hui Bae https://orcid.org/0000-0002-0342-1439

\section{REFERENCES}

1. Rath E, Skrede S, Mylvaganam H, Bruun T. Aetiology and clinical features of facial cellulitis: a prospective study. Infect Dis (Lond) 2018;50:27-34.

2. Bonnetblanc JM, Bedane C. Erysipelas: recognition and management. Am J Clin Dermatol 2003;4:157-63.

3. French H, Schaefer N, Keijzers G, Barison D, Olson S. Intracranial subdural empyema: a 10-year case series. Ochsner J 2014;14:188-94.

4. Khatri IA, Wasay M. Septic cerebral venous sinus thrombosis. J
Neurol Sci 2016;362:221-7.

5. DiNubile MJ. Septic thrombosis of the cavernous sinuses. Arch Neurol 1988;45:567-72.

6. Southwick FS, Richardson EP Jr, Swartz MN. Septic thrombosis of the dural venous sinuses. Medicine (Baltimore) 1986;65: $82-106$.

7. Priola SM, Moghaddamjou A, Ku JC, Taslimi S, Yang VX. Acupuncture-induced cranial epidural abscess: case report and review of the literature. World Neurosurg 2019;125:519-26.

8. Widdrington JD, Bond H, Schwab U, Price DA, Schmid ML, McCarron B, et al. Pyogenic brain abscess and subdural empyema: presentation, management, and factors predicting outcome. Infection 2018;46:785-92.

9. Chughtai KA, Nemer OP, Kessler AT, Bhatt AA. Post-operative complications of craniotomy and craniectomy. Emerg Radiol 2019;26:99-107.

10. Traficante D, Riss A, Hochman S. Bifrontal brain abscesses secondary to orbital cellulitis and sinusitis extension. Int J Emerg Med 2016;9:23.

11. Brouwer MC, van de Beek D. Epidemiology, diagnosis, and treatment of brain abscesses. Curr Opin Infect Dis 2017;30:12934.

12. Constantin F, Niculescu PA, Petre O, Balasa D, Tunas A, Rusu I, et al. Orbital cellulitis and brain abscess: rare complications of maxillo-spheno-ethmoidal rhinosinusitis. Rom J Ophthalmol 2017;61:133-6.

13. Xu S, Wang L, Cooper E, Zhang M, Manheimer E, Berman B, et al. Adverse events of acupuncture: a systematic review of case reports. Evid Based Complement Alternat Med 2013;2013: 581203.

14. Ernst E, Lee MS, Choi TY. Acupuncture: does it alleviate pain and are there serious risks? A review of reviews. Pain 2011;152 755-64.

15. White A. A cumulative review of the range and incidence of significant adverse events associated with acupuncture. Acupunct Med 2004;22:122-33. 